\title{
Probiotics, Prebiotics and Postbiotics in Atopic Dermatitis
}

\author{
Enza D’Auria*, Simone Pilloni, Silvia Beretta, Laura Paradiso and GianVincenzo Zuccotti \\ Department of Pediatrics-Vittore Buzzi Children's Hospital-University of Milan, Italy
}

*Corresponding author: Enza D’Auria, Department of Pediatrics-Vittore Buzzi Children's Hospital-University of Milan, Italy

\begin{abstract}
ARTICLE INFO
Received: 击 November 05, 2019

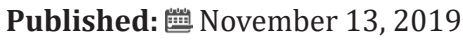

Citation: Enza D’Auria, Simone Pilloni, Silvia Beretta, Laura Paradiso, GianVincenzo Zuccotti. Probiotics, Prebiotics

\section{ABSTRACT}

Novel strategies are currently under investigation for the prevention and treatment of atopic dermatitis (AD). The supplementation of the diet with probiotics, prebiotics and postbiotics is probably one of the most promising approaches. Hereby, we summarize the current available evidence regarding the use of probiotics, prebiotics and postbiotics in AD. Biotic products seem to have a beneficial effect on AD, especially concerning its prevention. However, the evidence to support the supplementation of the diet with biotics for the management of $\mathrm{AD}$ is still not enough to make clear recommendations.
\end{abstract} and Postbiotics in Atopic Dermatitis. Biomed J Sci \& Tech Res 22(5)-2019. BJSTR. MS.ID.003804.
Keywords: Probiotics; Prebiotics; Postbiotics; Atopic Dermatitis; Microbiota

\section{Introduction}

'Atopic dermatitis (AD) is one of the most common chronic inflammatory disease of the skin. It affects about 15\%-30\% of children and $5-10 \%$ of adults. Although it most often starts in infancy, it may also develop during adolescence or adult life [1]. AD is characterized by dry skin, pruritus and eczematous skin lesions, that typically show age-related morphology and distribution [1,2]. Clinicians rely on clinical measures to assess the severity of disease and the outcomes of therapy. The most commonly used assessment tools include the SCORing Atopic Dermatitis (SCORAD) and Eczema Area and Severity Index (EASI) that have been validated to be used in both clinical settings and clinical trials [3]. AD has a profound impact on the quality of life of the patients and their families because of the burden of its complex signs and symptoms (itch, pain, sleep disturbance...) that affect social functioning and psychological well-being [4-6]. For these reasons, patients-reported symptoms and quality of life are also increasingly important in clinical practice $[7,8]$.

Actually, common therapeutic strategies for $\mathrm{AD}$ include hydration with emollients, avoidance of individual trigger factors, anti-inflammatory therapy with topical corticosteroids or calcineurin inhibitor and treatment of secondary infections [9-
11]. Increasing evidence shows that patients affected by $\mathrm{AD}$ have a modified composition of the gut microbiome and lack microbial diversity compared to healthy controls $[12,13]$. This bacterial dysbiosis may be considered a possible target for the treatment and prevention of AD. Therefore, the supplementation of the diet with probiotics, prebiotics and postbiotics may have a role in the management of $\mathrm{AD}$ because of their capacity of modulation of the microbiota.

The accepted definitions of probiotics, prebiotics and postbiotics are:

A. Probiotics: probiotics are defined as live microorganisms that, when administered in adequate amounts, confer a health benefit on the host [14];

B. Prebiotics: a prebiotic is a selectively fermented ingredient that allows specific changes, both in the composition and/or activity in the gastrointestinal microflora that confers benefits upon host wellbeing and health [15];

C. Postbiotics: postbiotics are bioactive compounds produced by food-grade microorganisms during a fermentation process. Postbiotics include microbial cells, cell constituents and 
metabolites [16]. In this short review we summarize the currently available evidence on the role of probiotics, prebiotics and postbiotics in the prevention and treatment of $\mathrm{AD}$.

\section{Probiotics in AD}

Probiotics are the most studied -biotic products for the prevention and treatment of AD. Most of the studies that evaluate the effectiveness of probiotics in the prevention of $\mathrm{AD}$ involve the intake of probiotics by the mother during pregnancy and the subsequent effect on infants and children. Concerning the prevention of $\mathrm{AD}$, the meta-analysis by Doege et al. [17], which included seven randomized, double-blind, placebo controlled clinical trials, assessed the impact of probiotics intake during pregnancy and lactation on the development of $\mathrm{AD}$ in children (2,843 total subjects included). The results showed that the administration of Lactobacilli during pregnancy could prevent AD in children aged 2-7 years, with a risk reduction of developing eczema of $10.6 \%$ $(\mathrm{p}=0.022)$. However, a mixture of various bacterial strains (with or without Lactobacilli) did not affect the development of AD [17]. The effect of probiotics supplementation on the prevention of AD was evaluated in another meta-analysis which included 14 studies.

The studies tested a variety of probiotic strains, the most commonly used was Lactobacillus rhamnosus GG, which was included in 6 of the 14 trials. The results of the meta-analysis indicated a $20 \%$ reduction in the incidence of $\mathrm{AD}$ in infants and young children following probiotic use, with a relative risk of atopic dermatitis of 0.79 ( $95 \%$ confidence interval $=0.71$ to 0.88 ). The effect on AD was similar according to the period of probiotic use (after delivery only or also during pregnancy) and the subjects receiving probiotics (mother, child, or both) [18]. A most recent meta-analysis which included 28 intervention trials $(6,705$ total participants) evaluated the effects of probiotic supplements on the development of atopic eczema in children. The probiotics were given either as single or multiple organisms in capsules, powder, or part of a drink or infant formula milk. This meta-analysis showed a reduction of atopic eczema at age $\leq 4$ years associated with the administration of probiotics (risk ratio $=0.78$, 95\% confidence interval $=0.65$ to 0.92 ).

The risk of eczema was reduced even more if the probiotics supplementation was administered to mothers during the postnatal period (risk ratio $=0.64,95 \%$ confidence interval $=0.51$ to 0.80 ) and not just to infants (risk ratio $=0.93,95 \%$ confidence interval $=0.81$ to 1.06) [19]. Lee etal. [20] conducted a meta-analysis which reviewed 19 randomized double-blind clinical trials assessing the prevention or treatment of AD in infants and children aged $0-13$ years $(1,898$ total subjects included). The results of the analysis favored the use of probiotics for prevention but not for treatment of AD. There was a significant risk reduction by as much as $61 \%$ associated with the use of prenatal and/or postnatal probiotics for the prevention of pediatric AD. Regarding the effect of probiotics supplementation on the treatment of $\mathrm{AD}$, the actual evidence is contrasting.
A recent meta-analysis, including 39 randomized-controlled trials $(2,599$ randomized participants), showed that currently available probiotic strains probably make little or no difference in improving patient-rated eczema symptoms (the symptom severity, on a scale from 0 to 20 , was 0.44 points lower after probiotic treatment), the quality of life for people with eczema and the investigator-rated eczema severity score (on a scale of 0 to 103 of the SCORAD index, the decrease was 3.91 points lower after probiotic treatment than after no probiotic treatment, while the minimum clinically important difference for SCORAD had been estimated to be 8.7 points). The probiotics used in the studies were bacteria of the Lactobacillus and Bifidobacterium species, either alone or combined with other probiotics, and were given with or without prebiotics [21]. Conversely, another meta-analysis included 7 double-blind randomized clinical trials (609 total subjects) evaluating the effect of probiotics on $\mathrm{AD}$ treatment in infants $\leq 36$ months.

Six studies used Lactobacillus containing preparations, while three used Bifidobacterium containing preparations. The metaanalysis indicated a beneficial effect of probiotics supplementation. The overall change in SCORAD index in infants was $-5.71(95 \%$ confidence interval $=-8.37$ to -3.04 , p value $<0.01$ ). The effect was especially protective in moderate-to-severe patients (overall change in SCORAD index=-8.32) and with preparations containing Lactobacillus (overall change in SCORAD index=-5.76) [22]. Huang et al. [23] included 13 randomized controlled studies (1,070 total subjects) in a recent meta-analysis. The data suggested an overall benefit of probiotics supplementation in children with $\mathrm{AD}$, indicated by a reduction of the SCORAD values of $-4.50(95 \%$ confidence interval=-7.45 to $-1.54, p$ value $<0.001$ ) in children aged 1-18 years. The beneficial effect was especially evident for Lactobacillus fermentum (mean difference $=-11.42$, 95\% confidence interval=-13.81 to -9.04$)$, Lactobacillus salivarius ( mean difference $=-7.21,95 \%$ confidence interval $=-9.63$ to -4.78 ) and a mixture of different strains (mean difference $=-3.52,95 \%$ confidence interval $=-5.61$ to -1.44 ).

\section{Prebiotics in AD}

The effect of prebiotics supplementation on the prevention and treatment of $\mathrm{AD}$ has been less studied than the effect of probiotics supplementation. One meta-analysis evaluated the effect of prebiotics supplementation on the prevention of allergy. The studies included in the meta-analysis assessed the effect of adding prebiotics (most commonly fructo-oligosaccharide, galactooligosaccharide and acidic oligosaccharides) to human milk or infant formula on the development of $\mathrm{AD}$ in children in the first six months of life. Four studies (1,428 total subjects) were included in the meta-analysis. The results indicated a significant reduction in eczema associated with the prebiotics supplementation (risk ratio $=0.68,95 \%$ confidence interval $=0.48$ to 0.97 ), suggesting that a prebiotic supplement added to infant foods may help to prevent the development of AD [24]. 
The meta-analysis by Garcia et al. [19] which included 10 intervention trials (4,242 total subjects) evaluating the effect of adding prebiotics (always a galacto-oligosaccharide, either alone or combined with other prebiotics or with probiotics) to the infant formula milk, did not show clear evidence that prebiotic supplementation reduces eczema at age $\leq 4$ years. Prebiotics and probiotics have also been combined in some studies in order to evaluate the benefit of the supplementation of this combination, defined as synbiotics, on the prevention and treatment of AD. The effects of synbiotics supplementation has been evaluated in a meta-analysis which included 6 treatment studies (369 total subjects) and 2 prevention studies (1,320 total subjects). The authors found a significant effect of synbiotics for the treatment of $\mathrm{AD}$ in children (weighted mean difference in SCORAD index of the synbiotics group compared with the control group $=-6.56,95 \%$ confidence interval $=-11.43$ to $-1.68, p$ value $=0.008$ ), especially when using mixed-strain probiotic components $(-7.32,95 \%$ confidence interval $=-13.98$ to -0.66 , $p$ value $=0.03$ ) and especially for children aged $\geq 1$ year $(-7.37,95 \%$ confidence interval $=-14.66$ to $-0.07, p$ value $=0.048$ ). The evidence for the use of synbiotics for the prevention of $\mathrm{AD}$ is not strong [25].

\section{Postbiotics in AD}

The evidence on the use of postbiotics for the prevention and/ or treatment of $\mathrm{AD}$ is still not consistent because this strategy has been proposed just in recent years. A pilot study investigating the clinical effects on $\mathrm{AD}$ of a supplementary diet prepared from rice flour fermented by Lactobacillus paracasei CBA L74 showed improvement in all patients. The SCORAD index of all the patients decreased after the supplementation (at week 12, all patients had a SCORAD index lower than 35), moreover all the patients reduced or suspended the application of topical steroids [26]. Another study evaluated the effect of supplementation of whey formula with viable or heat-inactivated Lactobacillus rhamnosus strain GG on the SCORAD scores in infants with AD. In this study, adverse gastrointestinal side effects were associated with the supplementation with heat-inactivated bacteria and not with the viable ones.

Atopic eczema and subjective symptoms improved significantly in both treatment groups, indeed the SCORAD index decreased from 15 (range $=0-29$ ) to 7 (range=0-26) units in the heat-inactivated LGG group [27]. The authors of another study investigated the clinical effect of a supplementary diet containing heat-killed Lactobacillus paracasei K71 on adult patients with AD. The skin severity scores significantly decreased in the group of patients taking postbiotics $(27.1 \%$ decrease at week $12, p$ value $<0.01)$ but not in the placebo group. The supplementation with heat-killed Lactobacillus paracasei K71 did not show evidence of influence on the itch scores or quality of life of the patients. The consumption of topical therapeutics decreased in the patients taking the postbiotics supplementation [28].

\section{Conclusion}

The supplementation of the diet with probiotics seems to have a beneficial role in $\mathrm{AD}$, especially regarding its prevention. Strong evidence is still lacking to recommend the use of probiotics in the treatment of AD, mostly due to the high heterogeneity of studies and strains used. In general, -biotic products seem to have a beneficial effect on AD. However, the evidence in favor of supplementing the diet with -biotics for the management of $\mathrm{AD}$ is still not enough to make clear recommendations.

\section{References}

1. Weidinger S, Novak N (2016) Atopic dermatitis. Lancet 387(10023): 1109-1122.

2. D’auria E, Banderali G, Barberi S, Gualandri L, Pietra B, et al. (2016) Atopic dermatitis: Recent insight on pathogenesis and novel therapeutic target. Asian Pacific J Allergy Immunol 34(2): 98-108.

3. Schmitt J, Langan S, Deckert S, Svensson A, Von Kobyletzki L, et al. (2013) Assessment of clinical signs of atopic dermatitis: A systematic review and recommendation. J Allergy Clin Immunol 132(6): 1337-1347.

4. Vakharia PP, Silverberg JI (2019) New and emerging therapies for paediatric atopic dermatitis. Lancet Child Adolesc Heal 3(5): 343-353.

5. Yang EJ, Beck KM, Sekhon S, Bhutani T, Koo J (2019) The impact of pediatric atopic dermatitis on families: A review. Pediatr Dermatol 36(1): 66-71.

6. D’Auria E, Barberi S, Cerri A, Boccardi D, Turati F, et al. (2017) Vitamin D status and body mass index in children with atopic dermatitis: A pilot study in Italian children. Immunol Lett 181: 31-35.

7. Chalmers JR, Simpson E, Apfelbacher CJ, Thomas KS, Von Kobyletzki L, et al. (2016) Report from the fourth international consensus meeting to harmonize core outcome measures for atopic eczema/dermatitis clinical trials (HOME initiative). Br J Dermatol 175(1): 69-79.

8. Boccardi D, D’Auria E, Turati F, Di Vito M, Sortino S, et al. (2017) Disease severity and quality of life in children with atopic dermatitis: POSCORAD in clinical practice. Minerva Pediatr 69(5): 373-380.

9. Wollenberg A, Barbarot S, Bieber T, Christen-Zaech S, Deleuran M, et al. (2018) Consensus-based European guidelines for treatment of atopic eczema (atopic dermatitis) in adults and children: part I. J Eur Acad Dermatology Venereol 32(5): 657-682.

10. Schneider L, Tilles S, Lio P, Boguniewicz M, Beck L, et al. (2013) Atopic dermatitis: A practice parameter update 2012. J Allergy Clin Immunol 131(2): 295-299.

11. Saini S, Pansare M (2019) New Insights and Treatments in Atopic Dermatitis. Pediatr Clin North Am 66(5): 1021-1033.

12. Kim J, Kim H (2019) Microbiome of the Skin and Gut in Atopic Dermatitis (AD): Understanding the Pathophysiology and Finding Novel Management Strategies. J Clin Med 8(4): 444.

13. Pascal M, Perez-Gordo M, Caballero T, Escribese MM, Lopez Longo MN, et al. (2018) Microbiome and Allergic Diseases. Front Immunol 9.

14. Hill C, Guarner F, Reid G, Gibson GR, Merenstein DJ, et al. (2014) Expert consensus document: The international scientific association for probiotics and prebiotics consensus statement on the scope and appropriate use of the term probiotic. Nat Rev Gastroenterol Hepatol 11: 506-514.

15. Gibson GR, Probert HM, Loo J Van, Rastall RA, Roberfroid MB (2004) Dietary modulation of the human colonic microbiota: updating the concept of prebiotics. Nutr Res Rev 17(2): 259-275.

16. Wegh, Geerlings, Knol, Roeselers, Belzer (2019) Postbiotics and Their Potential Applications in Early Life Nutrition and Beyond. Int J Mol Sci 20(19): 4673. 
17. Doege K, Grajecki D, Zyriax BC, Detinkina E, Zu Eulenburg C, et al. (2012) Impact of maternal supplementation with probiotics during pregnancy on atopic eczema in childhood-a meta-analysis. Br J Nutr 107(1): 1-6.

18. Pelucchi C, Chatenoud L, Turati F, Galeone C, Moja L (2012) Probiotics Supplementation During Pregnancy or Infancy for the Prevention of Atopic Dermatitis 23(3): 402-414.

19. Garcia-larsen V, Ierodiakonou D, Jarrold K, Cunha S, Chivinge J, et al. (2018) Diet during pregnancy and infancy and risk of allergic or autoimmune disease : A systematic review and meta-analysis. PLoS Med 15(2): e1002507.

20. Lee J, Seto D, Bielory L (2008) Meta-analysis of clinical trials of probiotics for prevention and treatment of pediatric atopic dermatitis. J Allergy Clin Immunol 121(1): 116-121.

21. Makrgeorgou A, Bath-hextall F, Murrel D, Tang M, Roberts A, et al. (2018) Probiotics for treating eczema(Review). Cochrane Database Syst Rev 11: CD006135.

22. Zhao M, Shen C, Ma L (2018) Treatment efficacy of probiotics on atopic dermatitis, zooming in on infants: a systematic review and metaanalysis. International Journal of Dermatology 57(6): 635-641.

ISSN: 2574-1241

DOI: 10.26717/BJSTR.2019.22.003804

Enza D’Auria. Biomed J Sci \& Tech Res

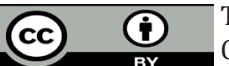

This work is licensed under Creative Commons Attribution 4.0 License

Submission Link: https://biomedres.us/submit-manuscript.php
23. Huang R, Ning H, Shen M, Li J, Zhang J (2017) Probiotics for the Treatment of Atopic Dermatitis in Children: A Systematic Review and Meta-Analysis of Randomized Controlled Trials. Front Cell Infect Microbiol 7: 392.

24. Osborn D, Sinn J (2013) Prebiotics in infants for prevention of allergy (Review). Cochrane Database Syst Rev 28(3): CD006474.

25. Chang Y, Trivedi MK, Jha A, Lin Y, Dimaano L, et al. (2016) Synbiotics for Prevention and Treatment of Atopic Dermatitis A Meta-analysis of Randomized Clinical Trials. JAMA Pediatr 170(3): 236-242.

26. Beretta S, Fabiano V, Petruzzi M, Budelli A, Zuccotti GV (2015) Fermented rice flour in pediatric atopic dermatitis. Dermatitis 26(2): 104-106.

27. Kirjavainen P V, Salminen SJ, Isolauri E (2003) Probiotic bacteria in the management of atopic disease: Underscoring the importance of viability. J Pediatr Gastroenterol Nutr 36(2): 223-227.

28. Moroi M, Uchi S, Nakamura K, Sato S, Shimizu N, et al. (2011) Beneficial effect of a diet containing heat-killed Lactobacillus paracasei K71 on adult type atopic dermatitis. J Dermatol 38(2): 131-139.

BIOMEDICAL
RESEARCHES $\quad$\begin{tabular}{l} 
Assets of Publishing with us \\
- Global archiving of articles \\
\hline
\end{tabular}

